\title{
Implementation of electromyography detecting system for exoskeleton robot applications
}

\begin{abstract}
In this study, Electromyography detecting circuit was implemented using high common mode rejection amplifier. The signal was detected from the skin of lower limb exactly from the Tibialis Anterior muscle below the knee during the ankle joint movement. This signal was collected using bipolar surface electromyography electrode after skin preparation procedure was carried out. The low noise, high precise instrumentation amplifier (INA114) was used in the first amplification process and the acquired signal was amplified with a gain of 100 and then fed to the passive band pass filter with (10ї 500) Hz cutoff frequencies. The filtered signal was amplified again in the second amplification stage using operational amplifier (OP2604AP) to get the signal in the volt range then displayed and stored on the digital oscilloscope. The displayed results still preliminary and more digital signal processing such as digital filtering, segmentation will be conducted in the future.
\end{abstract}

Keyword: Ankle joint movement; Electromyography; Signal conditioning 